\title{
Study of a Set of Symmetric Temporal Transformations for the Study of the Orbital Motion
}

\author{
José Antonio López Ortí (iD, Vicente Agost Gómez $\mathbb{D}^{\text {, }}$, and Miguel Barreda Rochera $\mathbb{C}$ \\ Department of Mathematics, Jaume I University, Castelló de la Plana, Castelló, Spain \\ Correspondence should be addressed to José Antonio López Ortí; lopez@mat.uji.es
}

Received 10 September 2021; Revised 1 November 2021; Accepted 23 November 2021; Published 13 February 2022

Academic Editor: Pritpal Singh

Copyright (c) 2022 José Antonio López Ortí et al. This is an open access article distributed under the Creative Commons Attribution License, which permits unrestricted use, distribution, and reproduction in any medium, provided the original work is properly cited.

\begin{abstract}
The main goal of this paper is to define a new one-parametric family of symmetric temporal transformations with respect to the ellipse. This new family contains as a particular case the eccentric anomaly, the regularized length of arc, and the elliptic anomaly. This family is a particular case of the biparametric family of anomalies introduced by the authors in 2016 . The biparametric family comprises the most common anomalies used in the study of the two-body problem. Two approaches of this work have been taken. The first one involves the study of the analytical properties of the symmetric family of anomalies. The second approach explores the improvement of the numerical integration methods when the natural time is replaced by an anomaly of this family.
\end{abstract}

\section{Introduction}

The two-body problem is a classical example of the integrability in celestial mechanics, which has already been studied by many authors [1-4] entre otros. This problem can be solved by means of a set of orbital elements $\vec{\sigma}$, known as the set III of Brower and Clemence [1], $(a, e, i, \Omega, \omega, M)$, where $a$ is the major semiaxis of the ellipse, $e$ is the eccenticity, $i$ is the true orbit inclination, $\Omega$ is the argument of the ascending node, $\omega$ is the argument of the periapsis, and $M$ is the mean anomaly for the epoch. Let $r, f$ be the polar coordinates of the secondary with respect to the primary in the orbital coordinate system $(\xi, \eta)$, where the origin is placed in the primary, the $\xi$ axis is running to the periapsis, and the $\eta$ axis is defined by the ellipse parameter position.

$$
\xi=r \cos f, \eta=r \sin f \text {. }
$$

The equation of the relative orbit is given by

$$
r=\frac{a\left(1-e^{2}\right)}{1+e \cos f} .
$$

The solution of the two-body problem can be reached through the eccentric anomaly, $g$. The mean anomaly, $M$, is connected with the eccentric anomaly, $g$, through the Kepler equation

$$
M=g-e \sin g .
$$

The orbital coordinates of the-two body problem can be described through the eccentric anomaly as

$$
\xi=a(\cos g-e), \eta=a \sqrt{1-e^{2}} \sin g, r=a(1-e \cos g) .
$$

The position of the secondary in the spatial system of coordinates $(x, y, x)$ is given by

$$
\left[\begin{array}{l}
x \\
y \\
z
\end{array}\right]=R_{1}(-\Omega) R_{3}(-i) R_{1}(-\omega)\left[\begin{array}{c}
a(\cos g-e) \\
a \sqrt{1-e^{2}} \sin g \\
0
\end{array}\right],
$$

where $R_{k}(\alpha)$ denotes the rotation matrix of angle $\alpha$ around the $k$ - axis. 
The perturbed motion can be solved by both analytical or numerical methods. The analytical methods are based on the perturbation method, where the zero-order solution is given by the two-body solution. The solution to the perturbed problem is reached by replacing the constant orbital elements by the osculator elements $(a(t), e(t), i(t), \Omega(t), \omega$ $(t), \sigma(t)), \sigma=M-\int_{t_{0}}^{t} n d t$, where $n$ is the mean motion given by the third Kepler law $a^{3} n^{2}=\mu$.

These quantities satisfy the Lagrange planetary equations $[1,3-5]$

$$
\begin{aligned}
\frac{d a}{d t} & =\frac{2}{n a} \frac{\partial R}{\partial \sigma}, \\
\frac{d e}{d t} & =-\frac{\sqrt{1-e^{2}}}{n a^{2} e} \frac{\partial R}{\partial \omega}+\frac{1-e^{2}}{n a^{2} e} \frac{\partial R}{\partial \sigma}, \\
\frac{d i}{d t} & =-\frac{1}{n a^{2} \sqrt{1-e^{2}} \sin i} \frac{\partial R}{\partial \Omega}+\frac{\operatorname{ctg} i}{n a^{2} \sqrt{1-e^{2}}} \frac{\partial R}{\partial \omega}, \\
\frac{d \Omega}{d t} & =\frac{1}{n a^{2} \sqrt{1-e^{2}} \sin i} \frac{\partial R}{\partial i}, \\
\frac{d \omega}{d t} & =\frac{\sqrt{1-e^{2}}}{n a^{2} e} \frac{\partial R}{\partial e}-\frac{\cos i}{n a^{2} \sqrt{1-e^{2}} \sin i} \frac{\partial R}{\partial i}, \\
\frac{d \sigma}{d t} & =-\frac{2}{n a} \frac{\partial R}{\partial a}-\frac{1-e^{2}}{n a^{2} e} \frac{\partial R}{\partial e},
\end{aligned}
$$

where $R$ is the perturbative potential. To integrate the Lagrange planetary equations by analytical methods, it is necessary to develop the second members of (6-11) as Fourier series using as temporal variable some kind of anomalies. The classical methods use the mean anomalies of the secondary and the disturbing bodies as the argument of the Fourier series. This technique is appropriate when the eccentricity of the motion is small, which is the case of planetary motion in the Solar System.

The numerical methods make a general study of the problem in the form

$$
\ddot{\vec{r}}=-\mu \frac{\vec{r}}{r^{3}}+\vec{F}
$$

where the first term represents the attraction of the primary and $\vec{F}$ denotes the perturbative forces. In general, the perturbative forces are small with respect to the attraction of the primary.

One of the main problems in the numerical methods is the nonuniform distribution of the points on the orbit when the natural time (or the mean anomaly) is used as temporal variable. In this case, the point distribution is concentrated in the apoapsis region and reaches its minimum in the periapsis region, which is contrary to the dynamics of the system. To solve this problem, we can follow several procedures

(i) To use of a very small integration step size

(ii) To use a variable step-size integrator (iii) To use an appropriate temporal transformation in order to improve the point distribution on the orbit

The first procedure is not convenient. The second one has been used profusely with very good results. The third procedure has been introduced, among others, by Hansen, Gilden [4], and improves integration results. This paper is focused on the third procedure, and this method can be combined with symplectic and variable step-size integrators.

The idea of a change in the temporal variable was introduced by Hansen through the partial anomalies [6]. Hansen defined two new variables to be used depending of the orbital region occupied by the secondary. The use of these anomalies improves the convergence of the analytical developments.

Sundman (1912) [7], in order to regularize the origin in the three-body problem, introduced a new temporal variable, $\tau$, connected with the time, $t$, through the transformation

$$
d t=\operatorname{Crd} \tau \text {. }
$$

Nacozy [7] introduced a new temporal variable called intermediate anomaly as

$$
d t=C r^{3 / 2} d \tau
$$

Janin and Bond $[8,9]$ defined a new one-parametric family of temporal variables called generalized Sundman anomalies

$$
d t=C_{\alpha} r^{\alpha} d \tau_{\alpha}
$$

These transformations can be described as

$$
d t=Q(r, \alpha) d \tau_{\alpha}
$$

where $Q(r, \alpha)$ is called as partition function. The mean $M$, eccentric $g$, and true $f$ anomalies are particular cases of the generalized Sundman anomalies family for $\alpha=0, \alpha=1$, and $\alpha=2$, respectively.

The regularized length of arc introduced by Brumberg [10] is related to the mean anomaly by

$$
Q(r)=\frac{1}{\sqrt{2 \mu}} \frac{\sqrt{r}}{\sqrt{1-r / 2 a}} .
$$

Brumberg and Fukushima [11] introduced the elliptic anomaly

$$
\omega=\frac{\pi u}{2 K(e)}-\frac{\pi}{2}, \operatorname{am} u=E+\frac{\pi}{2}
$$

where am $u$ is the elliptic amplitude of Jacobi and $K(e)$ the complete elliptic integral of the first kind. In this case, the partition function is given by

$$
Q(r)=\frac{1}{K(e) a^{2}} r^{3 / 2}\left(r^{\prime}\right)^{1 / 2} \text {, }
$$

where $K(e)$ is the complete elliptic integral of the first 
kind and $r^{\prime}=2 \mathrm{a}-r$. The regularized length of arc and the elliptic anomalies cannot be included in the generalized Sundman anomalies family. To solve this problem, López [12] defined a biparametric family of anomalies as

$$
C_{\alpha, \beta} r^{\alpha}\left(r^{\prime}\right)^{\beta} d \Psi_{\alpha, \beta}=d M
$$

where $r$ is the radius of the secondary with respect to the primary, and $r^{\prime}$ represents the distance between the secondary and the secondary focus of the ellipse. The biparametric family contains the regularized length of arc and the elliptic anomaly. The partition function can be written in the case of the regularized length of arc as

$$
Q(r)=\sqrt{2 h} r^{1 / 2}\left(r^{\prime}\right)^{-1 / 2},
$$

where $h$ is the integral of the energy.

The biparametric family contains these anomalies for $\alpha$ $=1 / 2$ and $\beta=-1 / 2$ in the case of regularized length of arc and $\alpha=3 / 2$ and $\beta=1 / 2$ for the elliptic anomaly.

The eccentric anomaly, the regularized length of arc, and the elliptic anomalies have a symmetric point distribution on the ellipse and satisfy the relation $\alpha-\beta=1$. In this paper, we study the biparametric family for the particular case $\alpha-\beta$ $=1$. This case is a one-parametric family of symmetric anomalies which contains the eccentric, the regularized lenght of arc, and the elliptic anomalies as particular cases.

In this article, we will study the properties of the family of anomalies and particularly a new anomaly introduced by López et al. [13], $\Psi$. This new anomaly is called the semifocal anomaly and is defined as the arithmetic mean between the angles comprised between the direction of the periapsis, and the secondary measured from the primary and secondary focuses of the ellipse, respectively. Thus

$$
\Psi=\frac{f+f^{\prime}}{2},
$$

where $f$ is the true anomaly, and $f^{\prime}$ is the antifocal anomaly introduced by Fukushima [14]; later, we will see that $f^{\prime}$ also belongs to the symmetric family of anomalies. In the first section of this paper, the background and the most common concepts have been introduced. Particularly, it is shown that a wide set of anomalies are part of the biparametric family introduced by the authors. It is also shown that some anomalies, namely, the ones with a symmetric point distribution, satisfy the relation $\alpha-\beta=1$.

In section two, a new anomaly has been defined as the mean between the true and the antifocal anomalies. We show that the main quantities involved in the two-body problem can be obtained in closed form when this anomaly is used. Also, we evince the properties of this anomaly revealing that it is included in the biparametric family, showing that it is symmetric with respect to both axes of the ellipse, and verifying the previous relation.
In section three, the most important developments for the symmetric family of anomalies have been obtained. First, the series are derived from the developments of $\Psi_{\alpha}$ as a function of $g$ when the relation $\alpha-\beta=1$ is satisfied in the biparametric family; if this condition holds, the anolamy is symmetric with respect of both axes of the ellipse. Second, applying Deprit's inversion algorithm on the development, we obtain all the developments needed in celestial mechanics.

In section four, a set of numerical examples are presented. We firstly observe that the point distribution along the orbit depends greatly on the choice of the anomaly. Following, we analyze the error committed in the integrations process; to that end, we use simple method applied to a high eccentric orbiting satellite after one revolution. The optimal value of the parameter $\alpha$ has been computed depending on the eccentricity. Finally, the local truncation errors along the orbit when natural time is used have been calculated.

In section five, the key conclusions and wider perspectives are presented.

\section{The Semifocal Anomaly}

Let us consider a new temporal variable, $\Psi$, defined as $\Psi=$ $\left(f+f^{\prime}\right) / 2$; this is the semifocal anomaly.

From Figure 1, it is easy to show that

$$
\begin{aligned}
\sin f & =\frac{a}{r} \sqrt{1-e^{2}} \sin g, \cos f=\frac{a}{r}(\cos g-e), \\
\sin f^{\prime} & =\frac{a}{r^{\prime}} \sqrt{1-e^{2}} \sin g, \cos f^{\prime}=\frac{a}{r^{\prime}}(\cos g-e),
\end{aligned}
$$

and from these equations, we have

$$
\begin{aligned}
& \sin (2 \Psi)=\sin \left(f+f^{\prime}\right)=\frac{a^{2}}{r r^{\prime}} \sqrt{1-e^{2}} \sin (2 g), \\
& \cos (2 \Psi)=\cos \left(f+f^{\prime}\right)=\frac{a^{2}}{r r^{\prime}}\left(\cos ^{2} g-\left(1-e^{2}\right) \sin ^{2} g\right),
\end{aligned}
$$

$$
1-\cos (2 \Psi)=2 \frac{a^{2}}{r r^{\prime}} \sin ^{2} g .
$$

To obtain the value of $\cos \Psi$ and $\sin \Psi$, we proceed as follows

$$
1+\cos (2 \Psi)=2 \frac{a^{2}}{r r^{\prime}}\left(1-e^{2}\right) \cos ^{2} g
$$

and from (27) and (28), we obtain

$$
\begin{aligned}
& \cos \Psi=\frac{\sqrt{1-e^{2}} \cos g}{\sqrt{1-e^{2} \cos ^{2} g},} \\
& \sin \Psi=\frac{\sin g}{\sqrt{1-e^{2} \cos ^{2} g}},
\end{aligned}
$$




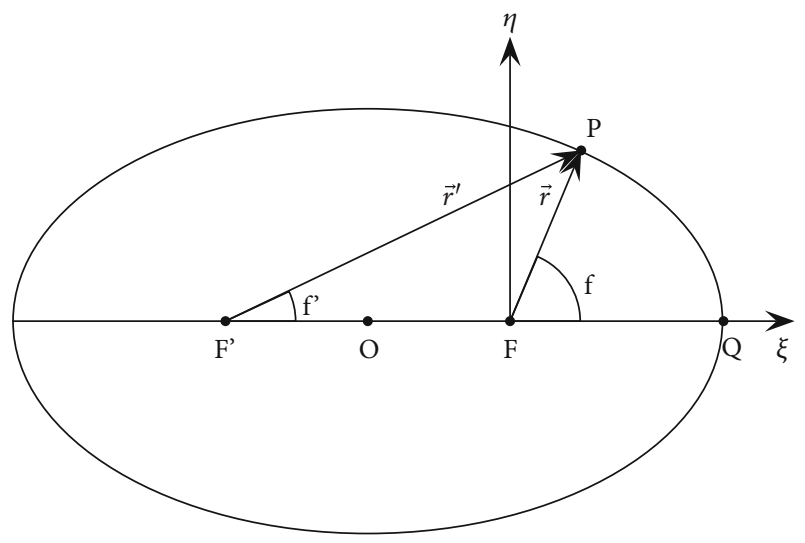

Figure 1: True and antifocal anomalies.

and so,

$$
\begin{gathered}
\cos g=\frac{\cos \Psi}{\sqrt{1-e^{2} \sin ^{2} \Psi}}, \\
\sin g=\frac{\sqrt{1-e^{2}} \sin \Psi}{\sqrt{1-e^{2} \sin ^{2} \Psi}} .
\end{gathered}
$$

From these equations, $\xi, \eta$, and $r$ can be related to $\Psi$ in a closed form.

Finally, for vector radius, we have

$$
r=a\left(1-\frac{e \cos \Psi}{\sqrt{1-e^{2} \sin ^{2} \Psi}}\right), r^{\prime}=a\left(1+\frac{e \cos \Psi}{\sqrt{1-e^{2} \sin ^{2} \Psi}}\right) .
$$

To obtain the partition function, we proceed as follows

$$
d \Psi=\frac{1}{2}\left(d f+d f^{\prime}\right)
$$

On the other hand, we have the well-known relation

$$
\frac{d f^{\prime}}{d f}=\frac{r}{r^{\prime}}
$$

and from them, we get

$$
d \Psi=\frac{1}{2}\left(1+\frac{r}{r^{\prime}}\right) d \mathrm{f}=\frac{r+r^{\prime}}{2 r^{\prime}} d f=\frac{a}{r^{\prime}} d f .
$$

Finally, taking into account the equation

$$
d f=\frac{a^{2}}{\sqrt{1-e^{2}}} r^{2} d M
$$

we obtain

$$
d M=Q\left(r, r^{\prime}\right) d \Psi
$$

where

$$
Q\left(r, r^{\prime}\right)=\frac{r^{2} r^{\prime}}{a^{3} \sqrt{1-e^{2}}}
$$

Thus, this anomaly can also be included in the biparametric family of anomalies with $\alpha=2$ and $\beta=1$. The semifocal anomaly satisfies the relation $\alpha-\beta=1$ and for this reason is considered a symmetric anomaly of the family defined above.

\section{Analytical Properties of the Symmetric Family of Anomalies}

In order to arrange the series development of the second members of the Lagrange planetary equations, we study a general method to obtain the Fourier series developments, according to an anomaly $\Psi_{\alpha}$, of the most common quantities in the two-body problem.

To this purpose, we obtain, in the first place, the value of the constant $C_{\alpha}$ that appears in (20) when $\alpha-\beta=1$. By differentiation Kepler's equation and taking into account the relation $\alpha-\beta=1$, we obtain

$$
C_{\alpha} d \Psi_{\alpha}=a^{-2 \alpha}(1-e \cos g)^{-\alpha+1}(1+e \cos g)^{-\alpha+1} d g
$$

Integrating $g$ and $\Psi_{\alpha}$ in $[0,2 \pi]$, we get

$$
C_{\alpha}=\frac{a^{-2 \alpha}}{2 \pi} \int_{0}^{2 \pi}\left(1-e^{2} \cos ^{2} g\right)^{-\alpha+1} d g
$$

and form this equation, we have

$$
d \Psi_{\alpha}=2 \pi K_{\alpha}\left(1-e^{2} \cos ^{2} g\right)^{-\alpha+1} d g
$$

where

$$
K_{\alpha}=\left[\int_{0}^{2 \pi}\left(1-e^{2} \cos ^{2} g\right)^{-\alpha+1} d g\right]^{-1}
$$

and $K_{\alpha}$ depends only of $e, \alpha$. This quantity can be expressed as a Taylor series with respect to the eccentricity. Next, we show the series up to tenth order in $e$ :

$$
\begin{aligned}
K_{\alpha}(e)= & +\left(\frac{1}{2}-\frac{\alpha}{2}\right) e^{2}+\left(\frac{1}{4}-\frac{5 \alpha}{16}+\frac{\alpha^{2}}{16}+\frac{\alpha^{3}}{96}\right) e^{4}+\left(\frac{1}{8}-\frac{13 \alpha}{96}+\frac{\alpha^{3}}{96}\right) e^{6} \\
& +\left(\frac{1}{32}-\frac{\alpha}{1536}-\frac{245 \alpha^{2}}{3072}+\frac{39 \alpha^{3}}{1024}+\frac{29 \alpha^{4}}{3072}+\frac{5 \alpha^{5}}{3072}\right) e^{10}
\end{aligned}
$$

In order to allow the development of $\Psi_{\alpha}$ according to $g$, we proceed as follows. First, let us define the new variable $z=\exp (\sqrt{(-1)} g)$ replacing $\cos \Psi_{\alpha}$ in the equation (51). We have

$$
d \Psi_{\alpha}=2 \pi K_{\alpha} G(e, z) d g
$$


where the generating function $G(z, e)$ is given by

$$
G(e, z)=\left(\frac{-e^{2} z^{4}+\left(4-2 e^{2}\right)-e^{2}}{4 z^{2}}\right)^{-\alpha+1},
$$

and so

$$
G(e, z)=\left(\frac{-e^{2}\left(z^{2}-z_{1}^{2}\right)\left(z^{2}-z_{2}^{2}\right)}{4 z^{2}}\right)^{-\alpha+1},
$$

where $z_{1}^{2} z_{2}^{2}=1$. The quantities $z_{1}=2-e^{2}+2 \sqrt{1-e^{2}} / e^{2}$ and $z_{2}=2-e^{2}-2 \sqrt{1-e^{2}} / e^{2}$ are the roots of the equation $G(z, e)=0$.

We can see that $\left.\left|z_{2}^{2}\right| \in\right] 0,1\left[,\left|z_{1}^{2}\right| \in\right] 1, \in \infty[$.

$$
G(e, z)=\frac{e^{2(1-\alpha)} z_{1}^{2(1-\alpha)}}{4^{2(1-\alpha)}}\left(1-\frac{z^{2}}{z_{1}^{2}}\right)\left(1-\frac{1}{z^{2} z_{1}^{2}}\right)
$$

Developing in series and after some algebraic manipulation, we obtain

$$
\begin{aligned}
d \Psi_{\alpha}= & 2 \pi K_{\alpha} \frac{e^{2(1-\alpha)} z_{1}^{2(1-\alpha)}}{4^{2(1-\alpha)}} \\
& \cdot\left[\sum_{k=0}^{\infty}\left(\begin{array}{c}
1-\alpha \\
k
\end{array}\right) \frac{1}{z_{1}^{2 k}}+2 \sum_{n=1}^{\infty}\left(\begin{array}{c}
1-\alpha \\
n+k
\end{array}\right) \frac{\cos (2 n g)}{z_{1}^{2 n}}\right] d g
\end{aligned}
$$

and integrating, we obtain a development in the form

$$
\Psi_{\alpha}=g+\sum_{k=1}^{\infty} S_{k}(\alpha, e) \sin (k g)
$$

where $S_{k}(\alpha, e)$ are analytical functions of order $k$ in $e$, and thus (50) can be reordered by powers of $e$.

The explicit form of the expansion of $\Psi_{\alpha}$ can be derived from (49) and (44). We obtain the development of $\Psi_{\alpha}$ according to $g$ and up to sixth order in $e$, we have

$$
\begin{aligned}
\Psi_{\alpha}= & g+\left(-\frac{1}{128} \alpha^{3} e^{6}+\frac{\alpha^{2} e^{6}}{32}+\frac{5 \alpha e^{6}}{128}+\frac{\alpha e^{4}}{8}+\frac{\alpha e^{2}}{4}-\frac{e^{6}}{16}-\frac{e^{4}}{8}-\frac{e^{2}}{4}\right) \\
& \cdot \sin (2 g)+\left(\frac{\alpha^{2} e^{6}}{64}+\frac{\alpha^{2} e^{4}}{64}-\frac{\alpha e^{6}}{64}-\frac{\alpha e^{4}}{64}\right) \sin (4 g) \\
& +\left(\frac{\alpha^{3} e^{6}}{1152}-\frac{\alpha e^{6}}{1152}\right) \sin (6 g) .
\end{aligned}
$$

From this main development, we can use Deprit algorithm to achieve the most common quantities involved in the two-body problem. From these developments, analytical theories of the motion can be formulated.
In the first place, we show the development of $\Psi_{\alpha}$ according $g$

$$
\begin{aligned}
g= & \Psi_{\alpha}+\left(\frac{3 \alpha^{3} e^{6}}{256}-\frac{3 \alpha^{2} e^{6}}{64}-\frac{5 \alpha e^{6}}{256}-\frac{\alpha e^{4}}{8}-\frac{\alpha e^{2}}{4}+\frac{7 e^{6}}{128}+\frac{e^{4}}{8}+\frac{e^{2}}{4}\right) \\
& \cdot \sin \left(2 \Psi_{\alpha}\right)+\left(\frac{3 \alpha^{2} e^{6}}{64}+\frac{3 \alpha^{2} e^{4}}{64}-\frac{7 \alpha e^{6}}{64}-\frac{7 \alpha e^{4}}{64}+\frac{e^{6}}{16}+\frac{e^{4}}{16}\right) \\
& \cdot \sin \left(4 \Psi_{\alpha}\right)+\left(-\frac{29 \alpha^{3} e^{6}}{2304}+\frac{3 \alpha^{2} e^{6}}{64}-\frac{133 \alpha e^{6}}{2304}+\frac{3 e^{6}}{128}\right) \sin \left(6 \Psi_{\alpha}\right) .
\end{aligned}
$$

Notice that the developments (51) and (52) contain only sinus of even arguments. $\Psi$ point distribution symmetry is the same as $g$. This fact induces the family simmetry on the ellipse.

To obtain the development of $\sin g$ and $\cos g$ according to $\Psi_{\alpha}$, we can use the Deprit inversion method [15], a Poisson series processor (PSP) [16], or a Mathematica package both developed by the authors. The source of the kernel of the PSP and the Mathematica package are available in the following URL: http://mecanicaceleste.uji.es; the source code of the PSP is free under General Public License v3.

The development of $\sin g$ is given by

$$
\begin{aligned}
\sin g= & \left(\frac{\alpha^{3} e^{6}}{256}-\frac{33 \alpha^{2} e^{6}}{1024}-\frac{\alpha^{2} e^{4}}{64}+\frac{7 \alpha e^{6}}{512}-\frac{\alpha e^{4}}{32}-\frac{\alpha e^{2}}{8}\right. \\
& \left.+\frac{15 e^{6}}{1024}+\frac{3 e^{4}}{64}+\frac{e^{2}}{8}+1\right) \sin \left(\Psi_{\alpha}\right) \\
& +\left(\frac{5 \alpha^{3} e^{6}}{512}-\frac{21 \alpha^{2} e^{6}}{1024}+\frac{\alpha^{2} e^{4}}{64}-\frac{9 \alpha e^{6}}{256}-\frac{13 \alpha e^{4}}{128}-\frac{\alpha e^{2}}{8}\right. \\
& \left.+\frac{47 e^{6}}{1024}+\frac{11 e^{4}}{128}+\frac{e^{2}}{8}\right) \sin \left(3 \Psi_{\alpha}\right)+\left(-\frac{17 \alpha^{3} e^{6}}{4608}+\frac{47 \alpha^{2} e^{6}}{1024}\right. \\
& \left.+\frac{\alpha^{2} e^{4}}{32}-\frac{103 \alpha e^{6}}{1152}-\frac{9 \alpha e^{4}}{128}+\frac{145 e^{6}}{3072}+\frac{5 e^{4}}{128}\right) \sin \left(5 \Psi_{\alpha}\right) \\
& +\left(-\frac{11 \alpha^{3} e^{6}}{1152}+\frac{35 \alpha^{2} e^{6}}{1024}-\frac{187 \alpha e^{6}}{4608}+\frac{49 e^{6}}{3072}\right) \sin \left(7 \Psi_{\alpha}\right) .
\end{aligned}
$$

For cos $g$, we have

$$
\begin{aligned}
\cos g= & \left(-\frac{1}{256} \alpha^{3} e^{6}+\frac{\alpha^{2} e^{6}}{1024}-\frac{\alpha^{2} e^{4}}{64}+\frac{25 \alpha e^{6}}{512}+\frac{3 \alpha e^{4}}{32}+\frac{\alpha e^{2}}{8}\right. \\
& \left.-\frac{47 e^{6}}{1024}-\frac{5 e^{4}}{64}-\frac{e^{2}}{8}+1\right) \cos \left(\Psi_{\alpha}\right) \\
& +\left(\frac{5 \alpha^{3} e^{6}}{512}-\frac{53 \alpha^{2} e^{6}}{1024}-\frac{\alpha^{2} e^{4}}{64}+\frac{11 \alpha e^{6}}{256}-\frac{3 \alpha e^{4}}{128}-\frac{\alpha e^{2}}{8}\right. \\
& \left.-\frac{e^{6}}{1024}+\frac{5 e^{4}}{128}+\frac{e^{2}}{8}\right) \cos \left(3 \Psi_{\alpha}\right) \\
& +\left(\frac{17 \alpha^{3} e^{6}}{4608}+\frac{17 \alpha^{2} e^{6}}{1024}+\frac{\alpha^{2} e^{4}}{32}-\frac{59 \alpha e^{6}}{1152}-\frac{9 \alpha e^{4}}{128}\right. \\
& \left.+\frac{95 e^{6}}{3072}+\frac{5 e^{4}}{128}\right) \cos \left(5 \Psi_{\alpha}\right)+\left(-\frac{11 \alpha^{3} e^{6}}{1152}+\frac{35 \alpha^{2} e^{6}}{1024}\right. \\
& \left.-\frac{187 \alpha e^{6}}{4608}+\frac{49 e^{6}}{3072}\right) \cos \left(7 \Psi_{\alpha}\right) .
\end{aligned}
$$




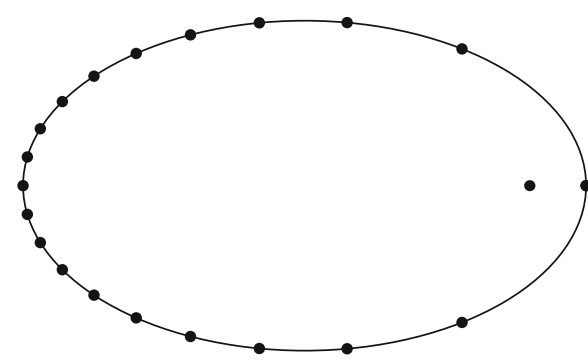

(a) $e=0.8, M$

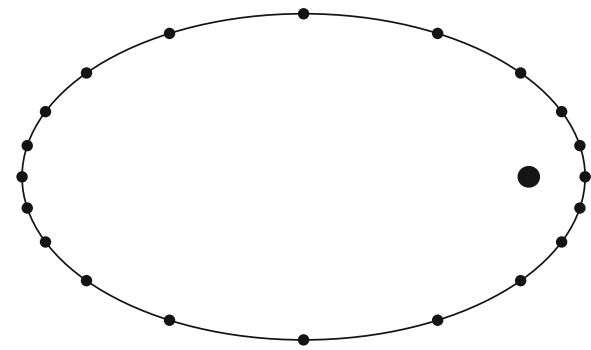

(c) $e=0.8, \Psi$

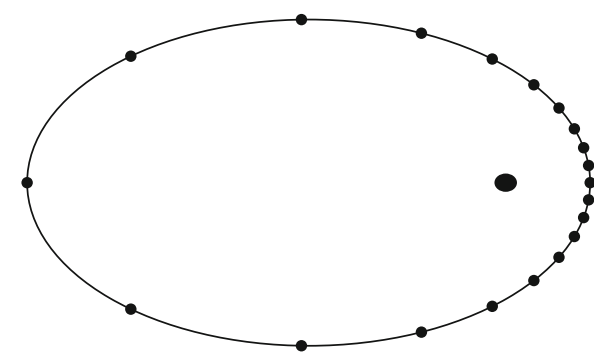

(b) $e=0.8, f$

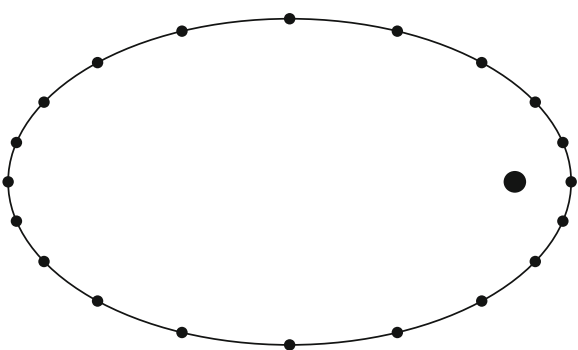

(d) $e=0.8, \omega$

FIgUre 2: Points distribution for $e=0.8$, and anomalies $M, f, \Psi, \omega$.

The Kepler equation for $\Psi_{\alpha}$ can be obtained from (52) and (53)

$$
\begin{aligned}
M= & \Psi_{\alpha}+\left(\frac{\alpha^{2} e^{5}}{64}+\frac{\alpha e^{5}}{32}+\frac{\alpha e^{3}}{8}-\frac{3 e^{5}}{64}-\frac{e^{3}}{8}-e\right) \sin \left(\Psi_{\alpha}\right) \\
& +\left(\frac{3 \alpha^{3} e^{6}}{256}-\frac{3 \alpha^{2} e^{6}}{64}-\frac{5 \alpha e^{6}}{256}-\frac{\alpha e^{4}}{8}-\frac{\alpha e^{2}}{4}+\frac{7 e^{6}}{128}+\frac{e^{4}}{8}+\frac{e^{2}}{4}\right) \\
& \cdot \sin \left(2 \Psi_{\alpha}\right)+\left(-\frac{1}{64} \alpha^{2} e^{5}+\frac{13 \alpha e^{5}}{128}+\frac{\alpha e^{3}}{8}-\frac{11 e^{5}}{128}-\frac{e^{3}}{8}\right) \\
& \cdot \sin \left(3 \Psi_{\alpha}\right)+\left(\frac{3 \alpha^{2} e^{6}}{64}+\frac{3 \Psi_{\alpha} \alpha^{2} e^{4}}{64}-\frac{7 \alpha e^{6}}{64}-\frac{7 \alpha e^{4}}{64}+\frac{e^{6}}{16}+\frac{e^{4}}{16}\right) \\
& \cdot \sin \left(4 \Psi_{\alpha}\right)+\left(-\frac{1}{32} \alpha^{2} e^{5}+\frac{9 \alpha e^{5}}{128}-\frac{5 e^{5}}{128}\right) \sin \left(5 \Psi_{\alpha}\right) \\
& +\left(-\frac{29 \alpha^{3} e^{6}}{2304}+\frac{3 \alpha^{2} e^{6}}{64}-\frac{133 \alpha e^{6}}{2304}+\frac{3 e^{6}}{128}\right) \sin \left(6 \Psi_{\alpha}\right) .
\end{aligned}
$$

Finally, to develop the second members of the Lagrange planetary equations, it is necessary to have the development of $r / a$ and $a / r$. The first quantity is given by

$$
\begin{aligned}
\frac{r}{a}= & +\left(\frac{\alpha^{2} e^{5}}{64}-\frac{3 \alpha e^{5}}{32}-\frac{\alpha e^{3}}{8}+\frac{5 e^{5}}{64}+\frac{e^{3}}{8}-e\right) \\
& \cdot \cos \left(\Psi_{\alpha}\right)+\left(\frac{\alpha^{2} e^{5}}{64}+\frac{3 \alpha e^{5}}{128}+\frac{\alpha e^{3}}{8}-\frac{5 e^{5}}{128}-\frac{e^{3}}{8}\right) \\
& \cdot \cos \left(3 \Psi_{\alpha}\right)+\left(-\frac{1}{32} \alpha^{2} e^{5}+\frac{9 \alpha e^{5}}{128}-\frac{5 e^{5}}{128}\right) \cos \left(5 \Psi_{\alpha}\right),
\end{aligned}
$$

and for $a / r$, we have

$$
\begin{aligned}
\frac{a}{r}=1 & +\frac{3 \alpha e^{6}}{16}+\frac{\alpha e^{4}}{8}+\frac{e^{6}}{8}+\frac{e^{4}}{4}+\frac{e^{2}}{2} \\
& +\left(-\frac{1}{64} \alpha^{2} e^{5}+\frac{9 \alpha e^{5}}{32}+\frac{\alpha e^{3}}{8}+\frac{23 e^{5}}{64}+\frac{5 e^{3}}{8}+e\right) \\
& \cdot \cos \left(\Psi_{\alpha}\right)+\left(-\frac{5}{128} \alpha^{2} e^{6}+\frac{19 \alpha e^{6}}{128}+\frac{23 e^{6}}{64}+\frac{e^{4}}{2}+\frac{e^{2}}{2}\right) \\
& \cdot \cos \left(2 \Psi_{\alpha}\right)+\left(-\frac{1}{64} \alpha^{2} e^{5}-\frac{15 \alpha e^{5}}{128}-\frac{\alpha e^{3}}{8}+\frac{57 e^{5}}{128}+\frac{3 e^{3}}{8}\right) \\
& \cdot \cos \left(3 \Psi_{\alpha}\right)+\left(-\frac{3 \alpha e^{6}}{16}-\frac{\alpha e^{4}}{8}+\frac{3 e^{6}}{8}+\frac{e^{4}}{4}\right) \cos (4 F) \\
& +\left(\frac{\alpha^{2} e^{5}}{32}-\frac{21 \alpha e^{5}}{128}+\frac{25 e^{5}}{128}\right) \cos \left(5 \Psi_{\alpha}\right) \\
& +\left(\frac{5 \alpha^{2} e^{6}}{128}-\frac{19 \alpha e^{6}}{128}+\frac{9 e^{6}}{64}\right) \cos \left(6 \Psi_{\alpha}\right) .
\end{aligned}
$$

\section{Numerical Examples}

In this section, we want to present a set of numerical examples in order to show the performance of the new symmetric family of variables introduced previously, focusing on the semifocal anomaly case. We desired the qualitative results to be orbit size independent, and to that end, the orbit has been parameterized with a specific anomaly. Thus, the temporal variable in the elliptic movement in comprised within the interval $[0,2 \pi]$. Taking into account these considerations, natural time must be replaced by the mean anomaly, M. 
TABLE 1: Integration errors in position $(\mathrm{km})$ and velocity $(\mathrm{km} / \mathrm{s})$ for several values of $\alpha$ symmetric family for the satellite Heos II.

\begin{tabular}{|c|c|c|c|c|c|}
\hline$\alpha$ & $\Delta \vec{r}$ & $|\Delta \vec{v}|$ & $\alpha$ & $\Delta \vec{r} \mid$ & $|\Delta \vec{v}|$ \\
\hline$M$ & $9.54 e+00$ & $7.71 e-03$ & 1.00 & $1.12 e-05$ & $9.08 e-09$ \\
\hline 0.00 & $1.51 e-02$ & $1.22 e-05$ & 1.05 & $1.21 e-05$ & $9.77 e-09$ \\
\hline 0.05 & $1.14 e-02$ & $9.21 e-06$ & 1.10 & $1.21 e-05$ & $9.79 e-09$ \\
\hline 0.10 & $8.40 e-03$ & $6.79 e-06$ & 1.15 & $1.16 e-05$ & $9.42 e-09$ \\
\hline 0.15 & $6.08 e-03$ & $4.91 e-06$ & 1.20 & $1.09 e-05$ & $8.83 e-09$ \\
\hline 0.20 & $4.34 e-03$ & $3.51 e-06$ & 1.25 & $1.00 e-05$ & $8.12 e-09$ \\
\hline 0.25 & $3.06 e-03$ & $2.47 e-06$ & 1.30 & $9.12 e-06$ & $7.38 e-09$ \\
\hline 0.30 & $2.14 e-03$ & $1.72 e-06$ & 1.35 & $8.20 e-06$ & $6.64 e-09$ \\
\hline 0.35 & $1.47 e-03$ & $1.19 e-06$ & 1.40 & $7.49 e-06$ & $6.06 e-09$ \\
\hline 0.40 & $1.01 e-03$ & $8.12 e-07$ & 1.45 & $6.77 e-06$ & $5.48 e-09$ \\
\hline 0.45 & $6.78 e-04$ & $5.48 e-07$ & 1.50 & $6.13 e-06$ & $4.96 e-09$ \\
\hline 0.50 & $4.51 e-04$ & $3.64 e-07$ & 1.55 & $5.67 e-06$ & $4.58 e-09$ \\
\hline 0.55 & $2.94 e-04$ & $2.38 e-07$ & 1.60 & $5.22 e-06$ & $4.22 e-09$ \\
\hline 0.60 & $1.88 e-04$ & $1.52 e-07$ & 1.65 & $4.83 e-06$ & $3.91 e-09$ \\
\hline 0.65 & $1.16 e-04$ & $9.34 e-08$ & 1.70 & $4.49 e-06$ & $3.63 e-09$ \\
\hline 0.70 & $6.78 e-05$ & $5.47 e-08$ & 1.75 & $4.32 e-06$ & $3.50 e-09$ \\
\hline 0.75 & $3.63 e-05$ & $2.92 e-08$ & 1.80 & $4.35 e-06$ & $3.52 e-09$ \\
\hline 0.80 & $1.59 e-05$ & $1.28 e-08$ & 1.85 & $4.27 e-06$ & $3.46 e-09$ \\
\hline 0.85 & $3.17 e-06$ & $2.51 e-09$ & 1.90 & $4.40 e-06$ & $3.57 e-09$ \\
\hline 0.90 & $4.56 e-06$ & $3.72 e-09$ & 1.95 & $4.74 e-06$ & $3.84 e-09$ \\
\hline 0.95 & $8.97 e-06$ & $7.28 e-09$ & 2.00 & $5.09 e-06$ & $4.13 e-09$ \\
\hline
\end{tabular}

First, we will show the point distribution along the orbit of the secondary with twenty points. These points are related to regular intervals of the chosen anomaly assuming the movement along a $e=0.8$ eccentricity ellipse.

In Figure 2, it can be seen that in the mean anomaly case, the points are gathered together around the apoapsis, leaving the periapsis zone with a very low point density. This fact is indeed a problem because in this zone the variation in the oscillation and the velocity with respect of the time are greater. Also in Figure 2, we can see that when the true anomaly, $f$, is used as the temporal variable, the point distribution is denser in the periapsis region and more scattered in the apoapsis. In both cases, we can observe that the point distribution geometry is symmetric with respect of the major axis of the ellipse, but not with respect of the minor axis. In the subfigures corresponding to the semifocal and elliptic anomalies, $\Psi$ and $\omega$, respectively, we notice a symmetric point distribution with respect of both axes of the ellipse; the density of the points is greater in the periapsis region and enormously enhances the concentration rate given by the mean anomaly.

Next, in order to be able to compare the results of this paper to those determined in previous publications, we are going to use the artificial satellite Heos II as our test bench. The orbital elements of this satellite, that was used before by Brumberg, are $a=118363.47 \mathrm{~km}, e=0.942572319, i=2$ $8^{\circ} .16096, \Omega=185^{\circ} .07554, \omega=270^{\circ} .07151$, and $M_{0}=0^{\circ}$. The period of the satellite is 4.69 days and the value of spaceflight constant for the Earth is $G M=3.986005 \mathrm{~km}^{3} \mathrm{~s}^{-2}$. Table 1 shows the error values of the position and velocity committed in the integration process with a classical 4thorder Runge-Kutta algorithm after one revolution and taking the semifocal anomaly as the temporal variable; the mean anomaly, $M$, has been firstly used and then the semifocal anomaly taking some values of the parameter.

Next, we study the value of the parameter that minimizes the error in the position after one revolution using several values of the parameter $\alpha$. Once again, a classical RungeKutta method with constant step size, $h=2 \pi / 1000$, has been chosen to evaluate the error. Table 2 shows the results.

Finally, we study the local truncation error when the mean and the semifocal anomalies are used as temporal variables. To that extent, we take 10000 evenly spaced points in $M$ and in $\Psi$ and we proceed by calculating the exact position in the $i$ node.

In the mean anomaly case, we solve the Kepler equation to obtain $g$, and from (4), we can attain the exact position $(\xi(i), \eta(i))$. This data is taken as an initial condition for the integration. We integrate with a classical fourth-order Runge-Kutta algorithm to obtain the approximation $(\widehat{\xi}(i+$ $1), \widehat{\eta}(i+1))$. This value is compared to the exact one for the $i+1$ node following the method previously described. 
TABle 2: Optimal values $\alpha$ for each value of $e$ in the symmetric family and optimal values and errors in position and velocity.

\begin{tabular}{|c|c|c|c|c|c|c|c|}
\hline$e$ & $\alpha$ & $\Delta \vec{r}$ & $\Delta \vec{v}$ & $e$ & $\alpha$ & $\Delta \vec{r}$ & $\Delta \vec{v}$ \\
\hline 0.000 & - & - & - & 0.500 & 0.130 & $1.0800 e-05$ & $1.0031 e-09$ \\
\hline 0.025 & 0.000 & $4.3170 e-05$ & $6.8668 e-10$ & 0.525 & 0.150 & $1.1491 e-05$ & $3.8381 e-10$ \\
\hline 0.050 & 0.000 & $4.7581 e-05$ & $7.7763 e-10$ & 0.550 & 0.220 & $1.1742 e-05$ & $5.6823 e-10$ \\
\hline 0.075 & 0.000 & $5.2481 e-05$ & $8.8259 e-10$ & 0.575 & 0.310 & $1.2406 e-05$ & $1.8567 e-09$ \\
\hline 0.100 & 0.000 & $5.7926 e-05$ & $1.0040 e-09$ & 0.600 & 0.360 & $1.4221 e-05$ & $2.3466 e-09$ \\
\hline 0.125 & 0.000 & $6.3985 e-05$ & $1.1450 e-09$ & 0.625 & 0.410 & $2.2682 e-05$ & $3.3040 e-09$ \\
\hline 0.150 & 0.000 & $6.7243 e-05$ & $1.1718 e-09$ & 0.650 & 0.450 & $2.1303 e-05$ & $3.9426 e-09$ \\
\hline 0.175 & 0.000 & $6.8698 e-05$ & $1.2378 e-09$ & 0.675 & 0.490 & $2.4100 e-05$ & $5.0445 e-09$ \\
\hline 0.200 & 0.000 & $6.9660 e-05$ & $1.3030 e-09$ & 0.700 & 0.530 & $3.5516 e-05$ & $7.1049 e-09$ \\
\hline 0.225 & 0.000 & $7.0063 e-05$ & $1.3663 e-09$ & 0.725 & 0.550 & $6.2882 e-05$ & $1.0223 e-09$ \\
\hline 0.250 & 0.000 & $6.9823 e-05$ & $1.4258 e-09$ & 0.750 & 0.600 & $3.3106 e-05$ & $1.1200 e-08$ \\
\hline 0.275 & 0.000 & $6.8832 e-05$ & $1.4790 e-09$ & 0.775 & 0.630 & $3.0239 e-05$ & $7.9479 e-09$ \\
\hline 0.300 & 0.000 & $6.6952 e-05$ & $1.5221 e-09$ & 0.800 & 0.670 & $6.9448 e-05$ & $2.4680 e-08$ \\
\hline 0.325 & 0.000 & $6.3986 e-05$ & $1.5494 e-09$ & 0.825 & 0.700 & $5.0922 e-05$ & $1.5274 e-08$ \\
\hline 0.350 & 0.000 & $5.9666 e-05$ & $1.5520 e-09$ & 0.850 & 0.740 & $2.9672 e-04$ & $9.3371 e-08$ \\
\hline 0.375 & 0.000 & $5.3600 e-05$ & $1.5165 e-09$ & 0.875 & 0.770 & $1.9490 e-05$ & $6.3435 e-08$ \\
\hline 0.400 & 0.000 & $4.5224 e-05$ & $1.4218 e-09$ & 0.900 & 0.800 & $1.3278 e-03$ & $3.6475 e-07$ \\
\hline 0.425 & 0.000 & $3.3715 e-05$ & $1.2346 e-09$ & 0.925 & 0.840 & $5.7060 e-04$ & $8.7792 e-08$ \\
\hline 0.450 & 0.000 & $1.7844 e-05$ & $9.0182 e-10$ & 0.950 & 0.880 & $2.1354 e-03$ & $2.7340 e-06$ \\
\hline 0.475 & 0.060 & $1.2287 e-05$ & $8.7805 e-10$ & 0.975 & 0.920 & $6.1986 e-02$ & $1.6956 e-04$ \\
\hline
\end{tabular}

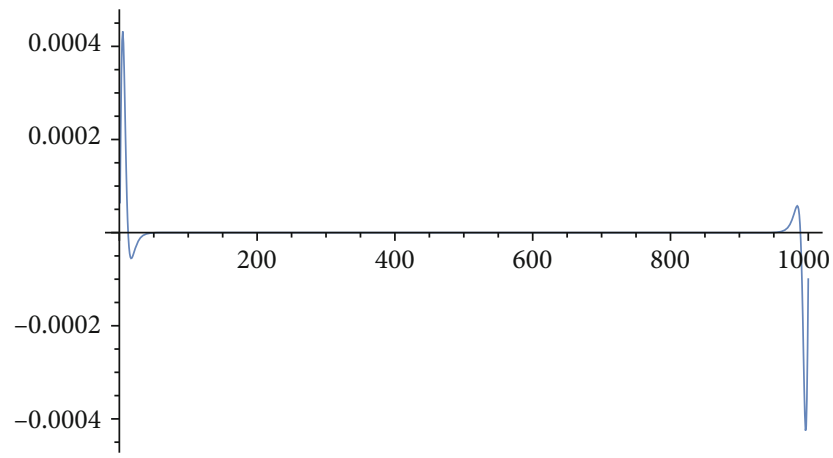

(a) errx in $\mathrm{km}$

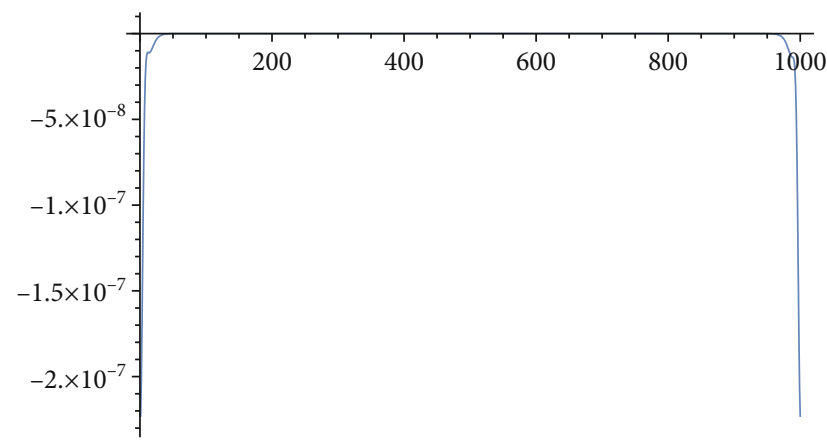

(c) errvx in $\mathrm{km} / \mathrm{s}$

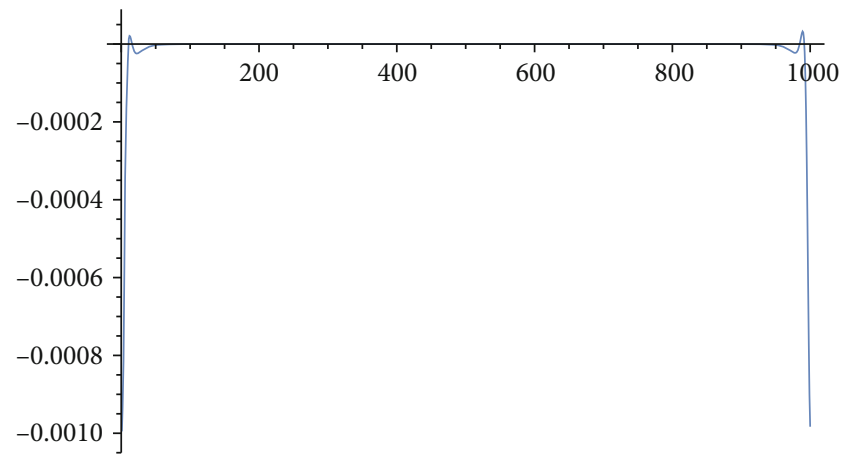

(b) erry in $\mathrm{km}$

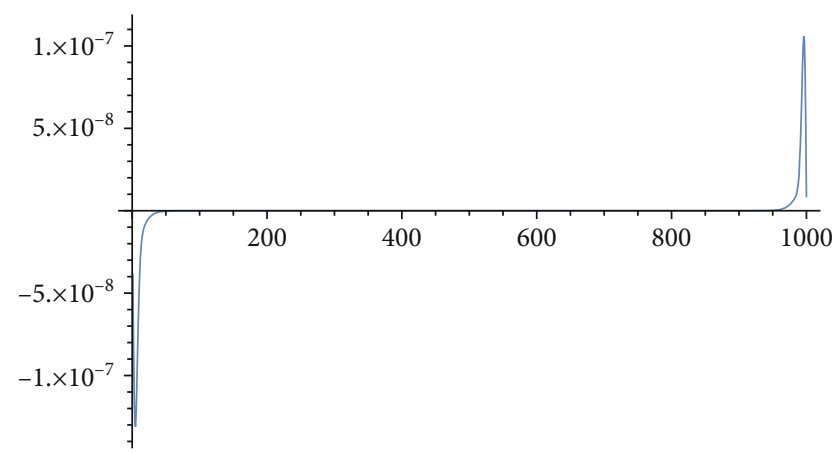

(d) errvx in $\mathrm{km} / \mathrm{s}$

Figure 3: Local truncation errors for $e=0.8$ and $M$. 


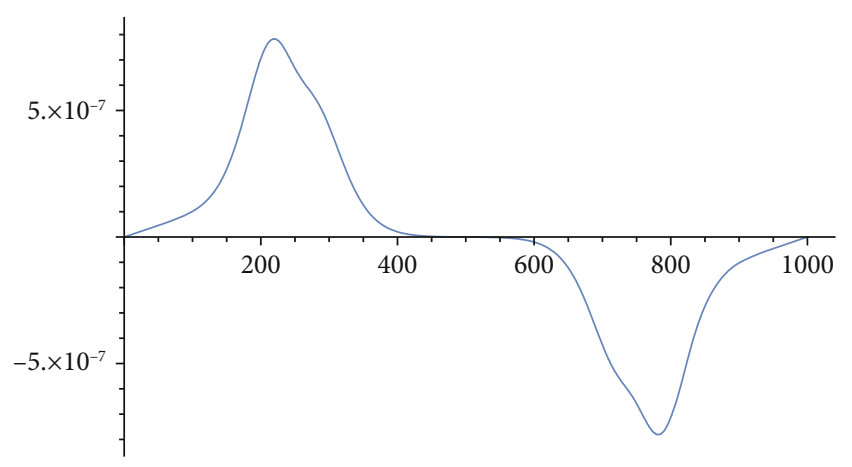

(a) errx in $\mathrm{km}$

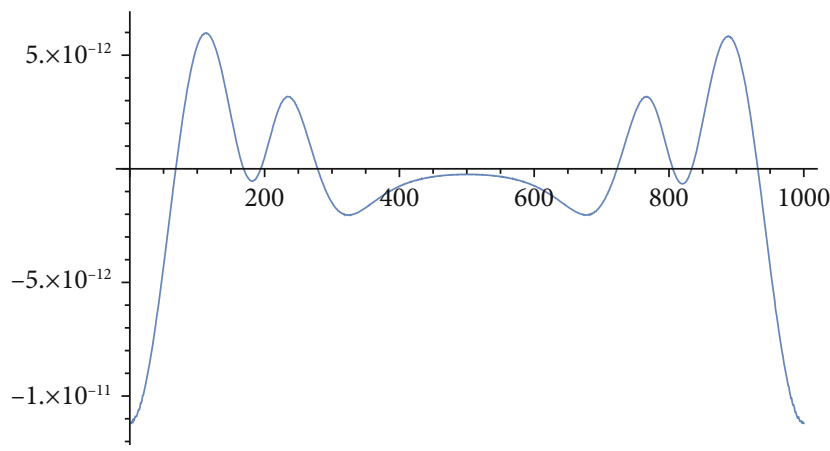

(c) errvx in $\mathrm{km} / \mathrm{s}$

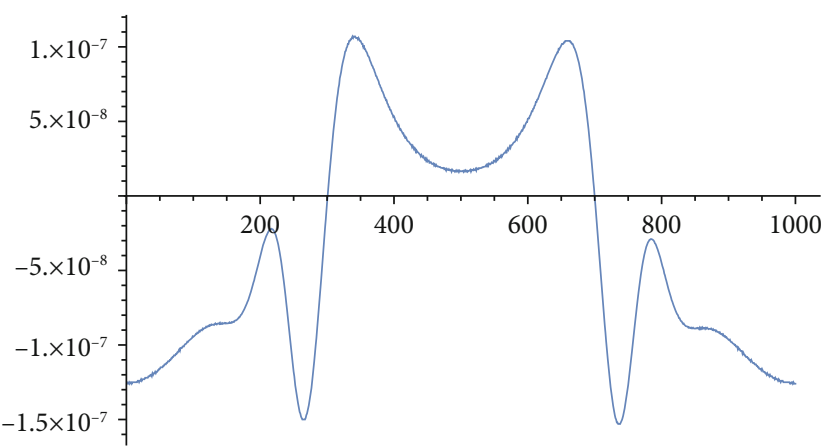

(b) erry in $\mathrm{km}$

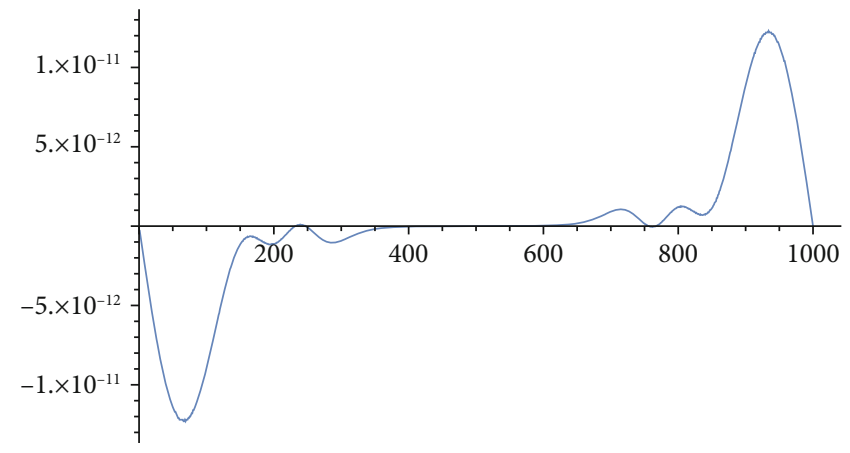

(d) errvx in $\mathrm{km} / \mathrm{s}$

Figure 4: Local truncation errors for $e=0.8$ and $\Psi$.

In the semifocal anomaly case, the exact value for the node $i$ can be obtained from the relations (31) and (32). This result replaced in (4) provides the exact value. Analogously proceeding as in $M$, we obtain the local truncation error distribution for the variable $\Psi$.

Figures 3 and 4 show the local truncation error connected to the variables $M$ and $\Psi$. We can see that the local truncation errors are considerably smaller when the semifocal anomaly is used instead of $M$.

\section{Concluding Remarks}

In this article, we first show that a set of variables-such as the eccentric or the elliptic anomaly-are encompassed within the biparametric family of anomalies defined by the authors. These anomalies provide a symmetric point distribution with respect of the major and minor semiaxes of the ellipse where the movement occurs. In the case of these anomalies, the relation $\alpha-\beta=1$ is always held.

Next, we introduce a new anomaly-the semifocal anomaly - as the mean between the true and the antifocal anomalies. This anomaly keeps a symmetric point distribution with respect of both axes and also presents interesting properties, considering that the main quantities of the twobody problem can be expressed in closed form; besides, the Kepler equation can also be expressed in closed form. At this point, we determine the partition function of this anomaly and we establish it is included in the biparametric family with the values $\alpha=2, \beta=1$. We define a set of symmetric anomalies within the biparametric family characterized by the relation $\alpha-\beta=1$.

When the eccentricity of the bodies that get involved in the movement around a given one with dominant mass is not high, their movements can be studied with perturbative techniques and analytical methods. To that extent, it is necessary to obtain the development as Fourier series of the most important magnitudes of the two-body problem as functions of the anomaly that is going to be used as temporal variable. In that sense, first, we obtain the development of the anomaly of the symmetric family as a function of the eccentric anomaly. With this, we have a development in which only even sines of the eccentric anomaly appear. This fact proves the symmetry of these anomalies with respect to both axes of the ellipse and justifies the symmetric anomalies' qualifier.

After that, applying Deprit inversion algorithm, we obtain the developments of $g, \sin g, \cos g, r / a$, and $a / r$ as Fourier series in the variable $\Psi_{\alpha}$. Thus, it is possible to obtain the developments of the second members of the Lagrange planetary equations according to these new variables.

Finally, we show the distribution of 20 evenly distributed points along the orbit using the mean anomaly, the true anomaly, the semifocal anomaly, and the elliptic anomaly, the last two as part of the symmetric family. Then, we provide some numerical examples to prove the efficiency of the use of these anomalies in the integration with numerical methods. This variable change is a new parametrization of the orbit, which implies it can be combined with other integration methods, such as symplectic integrators, variable step-size integrators, and extrapolation integrators. In this 
paper, we have chosen a simple constant step-size integrator since the objective of the previous section was to show the integration errors committed in one revolution of the high eccentric artificial satellite Heos II making use of several anomalies of this family. After that, we take a fictitious satellite and we show the same elements as in Eos II, but changing the eccentricity and looking for the value of the parameter $\alpha$ that minimizes the integration error. Lastly, we study the local truncation error of a fourth-order Runge-Kutta integrator along one orbital revolution; this study has been made for the mean anomaly and for the semifocal anomaly and for the latter the local truncation errors seam to be significantly enhanced.

In the future, we wish to extend this study to the hyperbolic case, where we hope to uncover interesting results.

\section{Data Availability}

No data were used to support this study.

\section{Conflicts of Interest}

The authors declare that they have no conflict of interest with regard to this work.

\section{Acknowledgments}

This work has been partially supported by grant 16I358.01/1 of University Jaume I of Castellón.

\section{References}

[1] D. Brower and G. M. Clemence, Celestial Mechanics, Ed Academic Press, New York, 1965.

[2] Y. Hagihara, Celestial Mechanics, vol. 2, MIT Press, Cambridge MA, 1970.

[3] J. Kovalevsky, Introduction to Celestial Mechanics, D. Reidel Publishing Company, DoDrecht-Holland, 1967.

[4] F. F. Tisserand, Traité de Mecanique Celeste, Gauthier-Villars, Ed., Paris, 1896.

[5] L. L. Levallois and J. Kovalewsky, Geodesie Generale, vol. 4, Eyrolles, Paris, 1971.

[6] P. Nacozy, "Hansen's method of partial anomalies: an application," The Astronomical Journal, vol. 74, pp. 544-550, 1969.

[7] P. Nacozy, "The intermediate anomaly," Celestial Mechanics, vol. 16, no. 3, pp. 309-313, 1977.

[8] G. Janin, "Accurate computation of highly eccentric satellite orbits," Celestial Mechanics, vol. 10, no. 4, pp. 451-467, 1974.

[9] G. Janin and V. R. Bond, The Elliptic Anomaly, NASA Technical Memorandum, 1980.

[10] E. V. Brumberg, "Length of arc as independent argument for highly eccentric orbits," Celestial Mechanics, vol. 53, no. 4, pp. 323-328, 1992.

[11] V. A. Brumberg and T. Fukushima, "Expansions of elliptic motion based on elliptic function theory," Celestial Mechanics, vol. 60, no. 1, pp. 69-89, 1994.
[12] J. A. López Ortí, V. Agost Gómez, and M. Barreda Rochera, “A new bi-parametric family of temporal transformations to improve the integration algorithms in the study of the orbital motion," Journal of Computational and Applied Mathematics, vol. 318, pp. 479-490, 2017.

[13] J. A. López, M. J. Martínez, and F. J. Marco, “A note on the use the semifocal anomaly as temporal variable in the two body problem," in Modelling for Engineering \& Human Behaviour 2020, pp. 87-90, Universidad Politécnica de Valencia, 2020.

[14] T. Fufkushima, "Efficient orbit integration by the orbital longitude method using antifocal anomaly," The Astronomical Journal., vol. 128, no. 3, pp. 1455-1464, 2004.

[15] A. Deprit, "Note on Lagrange's inversion formula," Celestial Mechanics, vol. 20, no. 4, pp. 325-327, 1979.

[16] J. A. López, V. Agost, and M. Barreda, "An improved C++ Poisson series processor with its applications," Computational and Mathematical Methods, vol. 14, article e1143, 2020. 